\title{
COMPARAÇÃO DE MÉTODOS E PROCESSOS DE AMOSTRAGEM PARA ESTUDOS FITOSSOCIOLÓGICOS EM UMA FLORESTA ECOTONAL NA REGIÃO NORTE MATOGROSSENSE
}

\author{
Jaime Antonio Ubialli ${ }^{1}$, Afonso Figueiredo Filho ${ }^{2}$, Sebastião do Amaral Machado ${ }^{3}$, \\ Julio Eduardo Arce ${ }^{3}$ \\ ${ }^{1}$ Eng. Florestal, Dr., Depto. de Eng. Florestal, UFMT, Cuiabá, MT, Brasil - jubialli@ yahoo.com.br \\ ${ }^{2}$ Eng. Florestal, Dr., Depto. de Engenharia Florestal, UNICENTRO, Irati, PR, Brasil - afig@ufpr.br \\ ${ }^{3}$ Eng. Florestal, Dr., Depto. de Ciências Florestais, UFPR, Curitiba, PR, Brasil - samachado@ufpr.br - jarce@ufpr.br
}

Recebido para publicação: 21/01/2008 - Aceito para publicação: 30/09/2008

\begin{abstract}
Resumo
O objetivo desta pesquisa foi caracterizar parametricamente a fitossociologia de uma floresta ecotonal de 120 hectares, situada na região norte matogrossense, bem como testar a acuracidade de processos, frações amostrais e métodos estimativos para oito grupos de espécies. Estudou-se a composição florística e sua diversidade, distribuição espacial de espécies e características da estrutura horizontal para árvores com DAP $\geq 30 \mathrm{~cm}$. Os processos aplicados foram aleatório e sistemático, com 5 e $10 \%$ de fração amostral, com 22 tamanhos e formas de parcelas variando de 400 a $10.000 \mathrm{~m}^{2}$. Identificaram-se 7.968 árvores distribuídas em 70 espécies (6 não identificadas), 58 gêneros e 31 famílias botânicas, apresentando, em média, 66,4 árvores e $11,08 \mathrm{~m}^{2}$ de área basal por hectare. A família botânica mais rica foi Fabaceae - Caesalpinioideae, e a mais frequente, Sapotaceae. A espécie mais importante foi Micropholis guyanensis (A. DC.) Pierre subsp. guyanensis. O padrão preponderante de distribuição das espécies é o aleatório nas parcelas de $400 \mathrm{~m}^{2}$ e agregado nas de $10.000 \mathrm{~m}^{2}$. Parcelas de 400 até $2.500 \mathrm{~m}^{2}$ estimaram com eficiência os valores de cobertura (VC) para o grupo das 15 espécies de maiores valores de importância (VI) e para as 6 comerciais mais importantes. Para os demais grupos de espécies, os erros mostram que os processos de amostragem aleatório e sistemático são inviáveis nas intensidades amostrais de 5 e $10 \%$.

Palavras-chave: Inventário florestal; censo florestal; processos amostrais; métodos amostrais; estrutura horizontal.
\end{abstract}

\begin{abstract}
Comparison of methods and processes of sampling for phitossociological studies of an ecotonal forest in the matogrosso northern region. The main objective of this research was to compare estimated phitossociological parameters obtained by sampling with true parameters obtained from the census of an ecotonal forest of 120 hectares, located in northern region of the Matogrosso State for eight groups of species. It was studied the floristic composition and the diversity, spatial distribution of species and characteristics of the horizontal structure for trees with $\mathrm{dbh} \geq 30 \mathrm{~cm}$. The applied sampling processes were random and systematic, with 5 and $10 \%$ of sampling fraction, with 22 sizes and forms of plots ranging from $400 \mathrm{~m}^{2}$ to $10,000 \mathrm{~m}^{2}$. It was identified 7,968 trees distributed in 70 species $(6$ not identified), 58 genera and 31 botanical families, presenting, in average, 66.4 trees and $11.08 \mathrm{~m}^{2}$ of basal area per hectare. The richest botanical family was Fabaceae - Caesalpinioideae and, the densest one was Sapotaceae. The most important species was Micropholis guyanensis (A.DC.) Pierre subsp. guyanensis. The preponderant standard of distribution of the species is the random one for the plot with $400 \mathrm{~m}^{2}$ and aggregate for the plots with $10,000 \mathrm{~m}^{2}$. Plots with 400 up to 2,500 $\mathrm{m}^{2}$ estimated with efficiency the values of covering for the group of the 15 commercial species with greatest VI (Importance value), and for the 6 most important ones. For the rest of the groups of species the errors showed that estimative processes (randon and systematic, with 5 and 10\%) are impracticable.

Keywords: Forest inventory; forest census; sampling processes; methods of sampling; horizontal Structure.
\end{abstract}




\section{INTRODUÇÃO}

Para que haja um aproveitamento racional e a conservação das florestas, são necessárias técnicas silviculturais adequadas, baseadas na ecologia de cada tipo de formação vegetal. A prescrição de técnicas adequadas necessárias para o aproveitamento permanente e sustentado implica o conhecimento da composição e da estrutura da floresta.

A análise estrutural fundamenta os critérios de colheita do plano de manejo florestal, permite avaliar o estágio de desenvolvimento da floresta e subsidia a aplicação de tratamentos silviculturais que promovem a melhoria da qualidade e produtividade da floresta.

A análise estrutural auxilia, sobretudo, a avaliação da efetividade da legislação florestal vigente para proteção e conservação dos recursos naturais (SILVA JÚNIOR, 2001), a valorização da floresta em pé (BENTES-GAMA et al., 2002), a elaboração de laudos periciais de desapropriação ambiental (ROCHA, 2003), o desenvolvimento de tecnologia para utilização de recursos florestais não-madeiráveis (FERREIRA; BRÁZ, 2004), os estudos de dinâmica das comunidades de florestas naturais (SOUZA et al., 2002 a,b; PAULA et al., 2002), a avaliação de impactos ambientais do manejo de florestas naturais (MARTINS et al., 2003), a avaliação de critérios e indicadores de sustentabilidade do manejo de florestas naturais (GOMES et al., 2004) e, de modo geral, a formulação e aplicação de ações ambientais que garantam a sustentabilidade de um projeto de base florestal.

Um adequado plano de manejo deve, portanto, ser a base para o aproveitamento racional dos recursos florestais existentes, para a conservação das florestas remanescentes e para se obterem subsídios necessários para a recuperação das florestas degradadas. Sua elaboração só é possível a partir da definição dos parâmetros de um ecossistema florestal, que devem ser fundamentados por um inventário florestal que, segundo Péllico Netto; Brena (1997), é uma atividade que visa obter informações qualitativas e quantitativas sobre os recursos florestais existentes em uma área pré-especificada.

O sucesso do inventário florestal está ligado à definição correta do processo de amostragem, do tamanho e forma das unidades amostrais e da intensidade da amostragem, requisitos básicos para a obtenção de informações com precisão.

Com relação ao processo amostral, Husch et al. (1972) definiram dois tipos de amostragem: com probabilidade e sem probabilidade, ou seja, aleatória ou sistemática. Husch (1971) ponderou que a amostragem aleatória elimina os erros sistemáticos ao eleger as unidades de amostra, além de permitir a quantificação do erro amostral, e que esses fatores se constituem na grande vantagem do processo aleatório em relação ao sistemático.

Por outro lado, Husch (1971) e Spurr (1952) afirmaram que na amostragem sistemática cada unidade amostral é mais bem distribuída espacialmente, isenta de tendência pessoal na sua escolha, menos onerosa na sua alocação e gera estimativas precisas da média e do total da população.

A intensidade de amostragem é definida no planejamento do inventário florestal e está intimamente relacionada ao conhecimento prévio da variação da população e da precisão pretendida (HIGUCHI et al., 1982).

Deve-se observar que a utilização de um processo de amostragem ocasiona a existência de um erro de amostragem, devido à medição de apenas parte da população e que quanto menor esse erro de amostragem, mais precisas são as estimativas obtidas. Portanto, a metodologia a ser desenvolvida no inventário, segundo Husch (1971), não deve ser baseada apenas na preferência do planejador, mas alicerçada em uma pesquisa científica específica visando determinar o tamanho e a forma que, para as condições da região em estudo, proporcionem as informações desejadas em um nível de precisão aceitável e com um mínimo de custo.

Diretamente ligada à adequação de tamanhos e formas de unidades de amostra, em florestas nativas, entre outros pesquisadores citam-se Silva (1980) em uma floresta tropical na região do Baixo Tapajós, no estado do Pará, Mello (1995) em um remanescente de floresta nativa no município de Lavras, Minas Gerais, e Bonetes (2003) na Floresta Nacional de Chapecó, no município de Guatambu, Santa Catarina.

Machado (1988), na Floresta Nacional do Tapajós (estado do Pará), realizou um inventário em 100\% de uma área de 3.012 hectares e comparou com os resultados obtidos na amostragem sistemática com conglomerados de $3.750 \mathrm{~m}^{2}$. Concluiu que o volume total e o número de árvores para todas as espécies foram muito próximos dos valores reais, gerando estimativas seguras por unidade de área. Todavia, as estimativas dessas variáveis por espécie podem levar a erros acentuados e fora do intervalo de confiança definidos em um inventário florestal. 
Bonetes (2003) testou a adequação de vários tamanhos e formas de unidades de amostra (variando de $200 \mathrm{~m}^{2}$ a $4.000 \mathrm{~m}^{2}$ ) para estimar o número de árvores e a área basal em uma Floresta Ombrófila Mista em Chapecó, estado de Santa Catarina, e concluiu que, para todas as espécies agrupadas, as estimativas foram muito precisas para todos os tamanhos e níveis amostrais testados. No entanto, quando os resultados foram comparados no nível de espécies, nenhum tamanho de parcela produziu estimativas confiáveis, tanto para estimar o estoque como para estudos fitossociológicos.

Dessa forma, o objetivo desta pesquisa foi testar a acuracidade de diversos tamanhos e formas de unidades de amostra, duas intensidades amostrais e dois processos de amostragem para estimar parâmetros fitossociológicos por espécie e grupos de espécies de uma Floresta Ecotonal na região norte matogrossense, comparando-os com os valores paramétricos obtidos pelo Censo.

\section{MATERIAIS E MÉTODOS}

\section{Área de estudo}

A área de estudo, de 120 hectares, localiza-se entre as coordenadas de paralelos $10^{\circ} 36^{\prime} 03^{\prime \prime} \mathrm{e}$

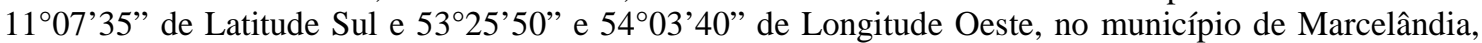
distante $891 \mathrm{~km}$ de Cuiabá, capital do estado de Mato Grosso, e é objeto de estudos pelo "Projeto de Manejo Sustentado para Usos Múltiplos da Floresta Tropical", da Faculdade de Engenharia Florestal da Universidade Federal de Mato Grosso.

De acordo com o levantamento fitogeográfico do Projeto RADAMBRASIL, Folha SC 22 Tocantins (BRASIL, 1981), encontra-se em uma área de tensão ecológica, na região de contato Floresta Ombrófila/Floresta Estacional, com predominância da formação denominada Floresta Semidecidual, Submontana, Dossel Emergente, com período sem chuvas de aproximadamente quatro a cinco meses, época em que, embora a maioria das espécies componentes do dossel arbóreo seja de árvores tipicamente amazônicas, cerca de $20 \%$ dos indivíduos perdem pelo menos parcialmente suas folhas, o que torna possível classificá-la como sendo Floresta Estacional.

Embasa-se em sedimentos do Terciário/Quaternário e se compõe de sedimentos arenopelitosos, predominantemente inconsolidados e parcialmente laterizados, com presença de cangas lateríticas. Geomorfologicamente localiza-se na unidade denominada Planalto dos Parecis e tem por característica relevo de formas tabulares, com topos aplanados separados por vales de fundo plano constituídos por solos do tipo Latossolo Vermelho/Amarelo distróficos, de textura média, que predominam associados a Latossolos Vermelho Escuro distróficos, de textura média e Areias Quartzosas distróficas.

De acordo com a classificação de Köppen, o clima dominante na região é do tipo AM (clima tropical monçoico), com precipitações médias entre 2250 e $2550 \mathrm{~mm} /$ ano, com período sem chuvas entre junho e agosto e chuvoso de janeiro a março. A temperatura média anual é de $28^{\circ} \mathrm{C}$.

\section{Coleta de informações}

$\mathrm{Na}$ área de 120 hectares $(1.000 \mathrm{~m} \times 1.200 \mathrm{~m})$, divididas em 20 faixas de $50 \mathrm{~m}$ no sentido da largura e $1.200 \mathrm{~m}$ de comprimento, com 6 hectares cada, foram medidas as variáveis de todos os indivíduos da população com diâmetros à altura do peito (DAP) iguais ou superiores a $30 \mathrm{~cm}$. As variáveis obtidas foram: nome vulgar da espécie, circunferência à altura do peito, altura comercial e as coordenadas de localização espacial (x,y) de cada árvore. Posteriormente foram classificadas, segundo seu aproveitamento, em espécies comerciais de uso regional em serraria, laminação e não comerciais.

As árvores, previamente identificadas no campo por seus nomes vulgares, foram classificadas em laboratório nos níveis de espécie, gênero e família, com base no Catálogo de Árvores do Brasil, editado pelo Instituto Brasileiro de Meio Ambiente e Recursos Naturais (IBAMA, 2001).

\section{Análises de dados}

Composição florística

A composição florística paramétrica (120 ha) foi expressa no nível de espécies, gêneros e famílias botânicas. Para a determinação da riqueza de espécies foi utilizado o quociente de mistura de Jentsch (BROWER; ZAR, 1984), e para a diversidade florística, o índice de Shannon (PIELOU, 1969, e MARTINS, 1993).

Padrão de distribuição espacial das espécies

Os padrões de distribuição das espécies, individualmente, na biocenose, e a comparação entre diferentes tamanhos de parcelas foram determinados pelos índices de agregação das espécies 
desenvolvidos por McGuiness e por Fracker e Brischle (BARROS; MACHADO, 1984) para unidades amostrais quadráticas de $400 \mathrm{~m}^{2}$ a $10.000 \mathrm{~m}^{2}$. Determinaram-se, parametricamente, as características das árvores de todas as espécies presentes que compuseram as parcelas citadas, pelo software Arc View 3.2. Para o processamento e a obtenção dos resultados finais, foi utilizado o programa FITOPAC 1, de Shepherd (1994).

Parâmetros da estrutural horizontal

As estatísticas paramétricas da estrutura horizontal (densidade, dominância e freqüências absolutas e relativas) e os valores de cobertura e de importância, citados por Daubenmire (1968), MuellerDombois e Ellenberg (1974), para todos os grupos de espécies, catalogadas em planilha EXCEL, foram determinadas pelo Programa "FLOREXEL", desenvolvido pelo Prof. Dr. Julio Eduardo Arce, vinculado ao Departamento de Ciências Florestais da UFPR. Os grupos de espécies definidos foram: todas as espécies, espécies com aproveitamento comercial, espécies comerciais para serraria, espécies comerciais para laminação, espécies de maior valor comercial, espécies não comerciais, 15 espécies de maior importância fitossociológica e espécie de maior valor de importância.

Tamanhos e formas de parcelas para estudos fitossociológicos

Foram utilizados 22 diferentes tamanhos de parcelas, variando de $400 \mathrm{~m}^{2}$ a $10.000 \mathrm{~m}^{2}$, de formas retangulares e quadráticas, muitas delas rotineiramente empregadas nos inventários florestais, por amostragem (Tabela 1).

Tabela 1. Dimensões das parcelas por tamanhos e formas.

Table 1. Plot dimensions by sizes and forms.

\begin{tabular}{|c|c|c|c|c|}
\hline \multirow{2}{*}{$\begin{array}{c}\text { Tamanho } \\
\left(\mathbf{m}^{2}\right)\end{array}$} & \multicolumn{4}{|c|}{ Forma } \\
\hline & Quadrada & & Retangular & \\
\hline 400 & $20 \mathrm{~m} \times 20 \mathrm{~m}$ & $40 \mathrm{~m} \times 10 \mathrm{~m}$ & & \\
\hline 500 & & & & $10 \mathrm{~m} \times 50 \mathrm{~m}$ \\
\hline 1000 & $32 \mathrm{~m} \times 32 \mathrm{~m}$ & $100 \mathrm{~m} \times 10 \mathrm{~m}$ & $50 \mathrm{~m} \times 20 \mathrm{~m}$ & $20 \mathrm{~m} \times 50 \mathrm{~m}$ \\
\hline 2000 & $45 \mathrm{~m} \mathrm{x} 45 \mathrm{~m}$ & $200 \mathrm{~m} \times 10 \mathrm{~m}$ & $100 \mathrm{~m} \times 20 \mathrm{~m}$ & $40 \mathrm{~m} \times 50 \mathrm{~m}$ \\
\hline 2500 & $50 \mathrm{~m} \times 50 \mathrm{~m}$ & $250 \mathrm{~m} \times 10 \mathrm{~m}$ & $125 \mathrm{~m} \times 20 \mathrm{~m}$ & \\
\hline 5000 & $71 \mathrm{~m} \times 71 \mathrm{~m}$ & $500 \mathrm{~m} \times 10 \mathrm{~m}$ & $250 \mathrm{~m} \times 20 \mathrm{~m}$ & $100 \mathrm{~m} \times 50 \mathrm{~m}$ \\
\hline 10000 & $100 \mathrm{~m} \times 100 \mathrm{~m}$ & $1.000 \mathrm{~m} \times 10 \mathrm{~m}$ & $500 \mathrm{~m} \times 20 \mathrm{~m}$ & $200 \mathrm{~m} \times 50 \mathrm{~m}$ \\
\hline
\end{tabular}

Os dados das variáveis de cada árvore foram determinados, para cada tamanho de parcela, pelo Programa Arc View 3.2 e transportados para a Planilha Excel, na qual os parâmetros e as estimativas foram determinados, para cada grupo de espécies trabalhadas e para cada uma das 704 simulações de unidades de amostra (Tabela 2). O processamento levou em conta ainda os dois processos de amostragem (aleatório e sistemático) e as duas intensidades amostrais (5 e 10\%).

Tabela 2. Desenhos amostrais simulados por processo e intensidade amostral.

Table 2. Simulated sampling designs by processes and by sampling intensity.

\begin{tabular}{lccccc}
\hline \multirow{2}{*}{ Processo } & \multicolumn{5}{c}{ Simulação da amostra } \\
\cline { 2 - 6 } & Intensidade (\%) & Parcelas & Grupos & Subtotal & Total \\
\hline \multirow{2}{*}{ Aleatório } & 5 & 22 & 8 & 176 & \\
& 10 & 22 & 8 & 176 & 352 \\
\multirow{2}{*}{ Sistemático } & 5 & 22 & 8 & 176 & \\
& 10 & 22 & 8 & 176 & 352 \\
\hline Total geral & & \multicolumn{5}{c}{} & 704 \\
\hline
\end{tabular}

A metodologia de seleção da amostra e as fórmulas matemáticas utilizadas para o processamento dos dados, considerando todos os grupos e desenhos amostrais testados neste trabalho, foram realizados como explicitado em Péllico Netto; Brena (1987). As análises acerca do desenho amostral mais adequado empregaram sempre o erro real, como também relatado em Péllico Netto; Brena (1987), e a variável considerada neste caso foi o valor de cobertura. 


\section{RESULTADOS E DISCUSSÕES}

\section{Composição florística}

$\mathrm{Na}$ área estudada foram identificadas 70 espécies (6 não foram identificadas) distribuídas em 56 gêneros e 30 famílias botânicas. A tabela 3 relaciona as espécies por família, nome científico, nome vulgar e seu aproveitamento comercial. As famílias Fabaceae, Apocynaceae e Sapotaceae compostas de 19, 5 e 4 espécies, respectivamente, são as mais representativas e agregam $40 \%$ das espécies encontradas nos 120 ha. Por outro lado, 15 famílias com apenas um gênero e uma espécie participam com somente $21,4 \%$ da riqueza da floresta.

Tabela 3. Espécies florestais encontradas na área e respectivos usos comerciais. Table 3. Forest species found in the area and respective commercial uses.

\begin{tabular}{|c|c|c|c|}
\hline Família & Nome científico & Nome vulgar & Uso(*) \\
\hline \multirow[t]{2}{*}{ Anacardiaceae } & Astronium gracile Engl. & Guarita & 3 \\
\hline & Tapirira guianensis Aubl. & Tapirira & 3 \\
\hline \multirow[t]{2}{*}{ Annonaceae } & Xylopia frutensis Aubl. & Pindaíba & 3 \\
\hline & Rollinia ennarginata Schletr. & Pinha-da-mata & 3 \\
\hline \multirow[t]{5}{*}{ Apocynaceae } & Aspidosperma nitidum Benth. Ex Mull.Arg & Guarantã & 3 \\
\hline & Sloanea sp. & Pateiro & 3 \\
\hline & Aspidosperma sp. & Peroba & 2 \\
\hline & Aspidosperma album (Vahl) R. Benoist ex Pichon & Peroba-mica & 2 \\
\hline & Himatanthus sucuuba (Spruce ex Müll. Arg.) Woodson & Sucuúba & 3 \\
\hline Araliaceae & Schefflera morototoni (Aubl.) Decne. \& Planch. & Mandiocão & 1 \\
\hline \multirow[t]{2}{*}{ Bignoniaceae } & Jacaranda copaia (Aubl.) D. Don & Caroba & 2 \\
\hline & Tabebuia sp. & Ipê & 2 \\
\hline \multirow[t]{3}{*}{ Burseraceae } & Protium heptaphyllum (Aubl.) March. & Amescla & 1 \\
\hline & Protium sp. & Amescla-breu & 2 \\
\hline & Trattinickia sp. & Morcegueira & $* * 1$ \\
\hline Celastraceae & Maytenus sp. & Espinheira-santa & 3 \\
\hline Clusiaceae & Callophyllum brasiliense Cambess. & Guanandi & 1 \\
\hline Combretaceae & Buchenavia sp. & Mirindiba & 2 \\
\hline Erythroxylaceae & Erythroxylum sp. & Marmelada & 3 \\
\hline Euphorbiaceae & Hevea sp. & Seringueira & 3 \\
\hline Fabaceae & Alexa grandiflora Ducke & Melancieiro & 1 \\
\hline \multirow[t]{8}{*}{ Fabaceae - Caesalpinioideae } & Cenostigma macrophyllum Tul. & Cascudo & 3 \\
\hline & Copaifera sp. & Copaíba & 2 \\
\hline & Apuleia leiocarpa (Vogel.) J. F. Macbr & Garapeira & 2 \\
\hline & Copaifera sp. & Guaranazinho & 3 \\
\hline & Hymenaea courbaril $\mathrm{L}$. var. courbaril & Jatobá & 2 \\
\hline & Myroxylon peruiferum L. F. & Pau-sangue & 3 \\
\hline & Dialium guianensis (Aulb.) Sandwith & Roxinho & 2 \\
\hline & Tachigali myrmecophila Ducke & Taxi-preto & 2 \\
\hline \multirow[t]{3}{*}{ Fabaceae - Faboideae } & Vouacapoua sp. & Angelim & 2 \\
\hline & Dipteryx odorata (Aubl.) Willd. & Cumbaru & $* * 2$ \\
\hline & Bowdichia nitida Spruce & Sucupira-preta & 2 \\
\hline \multirow[t]{7}{*}{ Fabaceae - Mimosoideae } & Dinizia excelsa Ducke & Angelim-pedra & 2 \\
\hline & Parkia pendula (Willd.) Benth. ex Walp. & Angelim-saia & 1 \\
\hline & Albizia hasslerii (Chodat) Burkart. & Farinha-seca & $* * 1$ \\
\hline & Inga sp. & Ingá & 3 \\
\hline & Pithecellobium montanum Benth. & Ingarana & 3 \\
\hline & Abarema jupunba (Willd.) Britton \& Killip & Saboeiro & 1 \\
\hline & Enterolobium maximum Ducke & Tamboril & 2 \\
\hline Goupiaceae & Goupia glabra Aubl. & Peroba-bosta & 2 \\
\hline
\end{tabular}

FLORESTA, Curitiba, PR, v. 39, n. 3, p. 511-523, jul./set. 2009. 


\begin{tabular}{|c|c|c|c|}
\hline \multirow[t]{3}{*}{ Lauraceae } & Ocotea sp. & Canela & 2 \\
\hline & Nectandra cuspidatea Nees & Canelão & $* * 2$ \\
\hline & Mezilaurus itauba (Meisn.) Tabu. ex Mez & Itaúba & $* * 2$ \\
\hline Lecythidaceae & Eschweilera micrantha $(\mathrm{O}$. Berg) Miers & Matamatá & 3 \\
\hline Lithraceae & Lafoensia pacari A. St.-Hil. & Amarelinho & 3 \\
\hline Malpighiaceae & $\begin{array}{l}\text { Byrsonima densa (Peir) DC. } \\
\text { Pseudobombax marginatum (A. St.-Hil., Juss. \& }\end{array}$ & Murici & 2 \\
\hline Malvaceae & Cambess.) A.Robyns & Sumaúma & 1 \\
\hline \multirow[t]{2}{*}{ Melastomataceae } & Mouriri sp. & Canela-de-cutia & 3 \\
\hline & Miconia sp. & Micônia & 3 \\
\hline \multirow[t]{3}{*}{ Moraceae } & Ficus pertusa L. F. & Figueira & 3 \\
\hline & Ficus insipida Willd & Gameleira & 1 \\
\hline & Brosimum sp. & L. brosimum & 3 \\
\hline \multirow[t]{2}{*}{ Myrtaceae } & Psidium sp. & Goiabinha & 3 \\
\hline & Eugenia sp. & Jambo & 3 \\
\hline Sapindaceae & Matayba guianensis Aubl. & Mataíba & 3 \\
\hline \multirow[t]{4}{*}{ Sapotaceae } & $\begin{array}{l}\text { Micropholis guyanensis (A. DC.) Pierre subsp. } \\
\text { guyanensis }\end{array}$ & Balata & 3 \\
\hline & Pouteria sp. & M.-da-mata & 3 \\
\hline & Micropholis melinoniana Pierre & Pau-de-sapo & 3 \\
\hline & Pouteria macrophylla (Lam.) Eyma & Taturuba & 3 \\
\hline Simaroubaceae & Simarouba amara Aubl. & Marupá & 1 \\
\hline Tiliaceae & Apeiba tibourbou Aubl. & Escova-de-macaco & 3 \\
\hline Urticaceae & Cecropia sp. & Imbaúba & 3 \\
\hline \multirow[t]{2}{*}{ Vochysiaceae } & Vochysia sp. & Cambará & $* * 2$ \\
\hline & Callisthene fasciculata Mart. & Carvoeiro & 3 \\
\hline
\end{tabular}

* Uso Comercial; 1: Laminação; 2: Serraria; 3: Não Comercial; **: Comerciais mais importantes

São 13 as famílias mais abundantes que, somadas às espécies não identificadas, com 396 indivíduos, representam 93,9\% das 7.968 árvores presentes na floresta (120 ha). Por outro lado, as 10 famílias menos representativas somam apenas 1,0\% das árvores. Leitão Filho (1987), em suas considerações sobre florística em 7 locais com florestas tropicais brasileiras, afirmou que em 5 desses trabalhos a família mais importante foi a Sapotaceae. Especialmente em florestas mesófilas semidecíduas, mencionou esse autor, há presença marcante de Fabaceae, Meliaceae, Rutaceae, Euphorbiaceae, Lauraceae e Myrtaceae, resultados bastante semelhantes aos encontrados nesta pesquisa.

$\mathrm{Na}$ área de estudo, 18 espécies têm frequências absolutas superiores a $1 \%$ do total, e as outras 52, consideradas raras, representam 74,3\% do total de espécies presentes na área. Segundo Martins (1993) e Kageyama; Gandara (1993), espécies raras são aquelas que se apresentam com menos de um indivíduo por hectare. É importante salientar que o limite de inclusão no presente estudo foi de $30 \mathrm{~cm}$ de DAP, e a definição desse limite interfere diretamente no conceito de espécies raras.

\section{Diversidade florística}

O quociente de mistura de espécies de Jentsch foi de 0,0088 e mostrou uma floresta bastante heterogênea e rica, com um número médio de indivíduos de 113,83 por espécie na área amostrada (120 ha), ressaltando-se o limite de inclusão considerado (DAP $\geq 30 \mathrm{~cm}$ ). O índice de Shannon (H') de 3,19 confirma a heterogeneidade florística da área. De acordo com Pielou (1975), esse índice varia de um até 3,5, raramente ultrapassando 4,5, e ratifica os valores obtidos na Floresta Ombrófila Mista por Longhi (1997), de 3,651, o de 3,084 por Guapyassu (1994) em uma Floresta Ombrófila Densa no Paraná e o detectado por Silva e Scariot (2004) em uma floresta estacional decidual em São Domingos (GO), de 3,18. Índices próximos foram obtidos em florestais tropicais de terra firme, segundo Martins (1979), por Black et al. (1950), em Tefé (AM) e Castanhal (PA), de 3,86 e 3,72, respectivamente, por Prince et al. (1976) em Manaus (AM), de 4,76, por Pires et al. (1953) em Castanhal (PA), de 4,3, por Bastos (1948) em Santa Maria de Vila Nova (AM), de 3,58, e por Rodrigues (apud Rizzini, 1979) em Serra do Navio (AM), de 3,89. 
Ressalte-se que os valores dos índices obtidos sofrem influência dos limites diamétricos (níveis de inclusão) com que as árvores foram coletadas e que não há padronização sobre os limites mínimos desses diâmetros.

\section{Padrão de distribuição das espécies}

Pelo índice de McGuiness para parcelas de $20 \mathrm{~m}$ x $20 \mathrm{~m}, 55,7 \%$ das espécies se distribuem aleatoriamente, $30,0 \%$ delas apresentam tendência ao agrupamento e $14,3 \%$ se distribuem uniformemente na área. Por outro lado, para as parcelas de $100 \mathrm{~m} \times 100 \mathrm{~m}, 47,1 \%$ se apresentam agrupadas, 40,0\% tendem ao agrupamento e 12,8\% estão uniformemente distribuídas. Já pelo índice proposto por Fracker e Brischle, $78,7 \%$ das espécies se distribuíram aleatoriamente nas parcelas menores, enquanto que apenas $21 \%$ apresentaram-se agrupadas ou com tendências ao agrupamento. Essa tendência se inverte nas parcelas maiores, demonstrando que $68,6 \%$ das espécies se agrupam, 18,6\% têm tendências a agrupar-se e apenas $12,8 \%$ distribuem-se aleatoriamente. Isso mostra que, na medida em que se aumenta o tamanho da parcela, há uma tendência de se ter um tamanho mais adequado, capaz de englobar o agrupamento das árvores pertencentes à mesma espécie, como descreveu Matteucci; Colma (1982).

Rondon Netto et al. (2000), em uma clareira de origem antrópica em Lavras, estado de Minas Gerais, descreve que o padrão predominante foi o agregado em $61,2 \%$ das espécies, aleatório em $36,7 \%$ e uniforme em $2,1 \%$. Esses resultados são mais próximos aos encontrados neste trabalho em unidades amostrais de maiores dimensões.

\section{Parâmetros da estrutura horizontal para todas as espécies}

As 70 espécies florestais encontradas na área com mais de $30 \mathrm{~cm}$ de DAP são responsáveis por 7.968 árvores, que representam um número médio de 66,4 árvores e $11,08 \mathrm{~m}^{2}$ de área basal por hectare. Três espécies (Micropholis guyanensis, Aspidosperma nitidum e Trattinickia sp.) participam com 4,3\% do total de espécies, $25,4 \%$ do valor de importância e 35,9\% do valor de cobertura de toda a área. Nessas espécies, acumulam-se 32,9\% da densidade e 38,9\% da dominância.

Micropholis guyanensis representa os maiores valores de importância e de cobertura por possuir a maior densidade, seguida pela Trattinickia sp. No entanto, a espécie Aspidosperma nitidum, apesar de menor abundância do que as duas primeiras, é responsável por mais de $15 \%$ de toda a área basal da floresta estudada (120 ha). As 20 espécies mais importantes somam 68,0\% da importância e 87,8\% do potencial produtivo. Elas detêm $87,3 \%$ das árvores e representam $88,2 \%$ de toda a cobertura horizontal da área. As outras 50, ou seja, 71,4\% das espécies participam com apenas $12,7 \%$ das árvores, $11,8 \%$ da área basal, $12,2 \%$ do valor de cobertura e $11,4 \%$ do total de espécies, ou seja, 8 delas possuem apenas uma árvore cada.

A espécie Micropholis guyanensis, conhecida vulgarmente por balata, é a mais importante fitossociologicamente, com um valor de importância e de cobertura médio de 9,2\% e 13,0\%, respectivamente. Apresenta a maior densidade relativa, $15,0 \%$, e a terceira maior dominância, com $11,1 \%$. Em termos absolutos, representa 9,91 indivíduos/ha e $1,23 \mathrm{~m}^{2}$ de área basal/há, aparecendo na área total 1.194 vezes. A tabela 4 apresenta os resultados obtidos para todos os parâmetros da estrutura horizontal da área. Mais detalhadamente, a estrutura paramétrica por espécies e por grupos de espécies, assim como as participações de cada grupo de espécies, pode ser observada em Ubialli (2007).

\section{Parâmetros da estrutura horizontal por grupos de espécies}

Como se observa na figura 1, as espécies comerciais apresentam menor densidade (DR) que as não comerciais, e esses valores se invertem para a dominância. São, no entanto, próximos para as 15 espécies de maior valor de importância (VI).

Para as frequências (FR), no entanto, o equilíbrio se mantém entre comerciais e não comerciais e se torna muito menor no grupo das 15 espécies de maior valor de importância. Isso não influencia no valor de cobertura (VC), mas é altamente determinante na diminuição do parâmetro valor de importância.

As 15 espécies fitossociologicamente mais importantes, representando $21,4 \%$ das espécies presentes na floresta, são responsáveis por $61,0 \%$ do valor de importância, $80,8 \%$ do valor de cobertura, $81,0 \%$ da densidade e por $80,7 \%$ da dominância (DoR) na área. Isso comprova que em poucas espécies se concentra a maior importância da estrutura horizontal da área. Dessas espécies, as mais importantes são a Micropholis guyanensis, a Aspidosperma nitidum e a Trattinickia sp. 
Tabela 4. Estrutura horizontal paramétrica.

Table 4. Parametric horizontal structure.

\begin{tabular}{lcccccc}
\hline Nome científico & DA & DR & DoR & FR & Vc\% & VI\% \\
\hline Micropholis guyanensis & 10,0 & 15,0 & 11,1 & 1,4 & 13,0 & 9,2 \\
Aspidosperma nitidum & 5,5 & 8,3 & 15,2 & 1,4 & 11,8 & 8,3 \\
Trattinickia sp. & 6,4 & 9,6 & 12,7 & 1,4 & 11,1 & 7,9 \\
Ocotea sp. & 4,3 & 6,5 & 4,5 & 1,4 & 5,5 & 4,2 \\
Vochysia sp. & 3,9 & 5,8 & 4,8 & 1,4 & 5,3 & 4,0 \\
Dialium guianense & 3,6 & 5,4 & 4,8 & 1,4 & 5,1 & 3,9 \\
Mezilaurus itauba & 2,2 & 3,3 & 6,2 & 1,4 & 4,8 & 3,7 \\
Brosimum sp. & 3,6 & 5,4 & 3,8 & 1,4 & 4,6 & 3,5 \\
Albizia hasslerii & 2,8 & 4,2 & 3,2 & 1,4 & 3,7 & 3,0 \\
Ni & 2,6 & 4,0 & 3,0 & 1,4 & 3,5 & 2,8 \\
Apeiba tibourbou & 2,1 & 3,1 & 3,5 & 1,4 & 3,3 & 2,7 \\
Miconia sp. & 2,5 & 3,8 & 2,5 & 1,4 & 3,2 & 2,6 \\
Inga sp. & 1,7 & 2,6 & 2,1 & 1,4 & 2,3 & 2,0 \\
Dipteryx odorata & 1,3 & 2,0 & 2,0 & 1,4 & 2,0 & 1,8 \\
Nectandra cuspidatea & 1,4 & 2,2 & 1,5 & 1,4 & 1,8 & 1,7 \\
Bowdichia nítida & 1,2 & 1,9 & 1,3 & 1,4 & 1,6 & 1,5 \\
15 espécies + Ni(6) & 55,2 & 83,1 & 82,2 & 22,9 & 82,7 & 62,7 \\
Outras (49 espécies) & 44,8 & 16,9 & 17,8 & 77,1 & 17,3 & 37,3 \\
\hline DA: Didi
\end{tabular}

DA: Densidade absoluta; DR: Densidade relativa; DoR: Dominância relativa; FR: Frequência relativa; VC: Valor de cobertura; VI: Valor de importância.

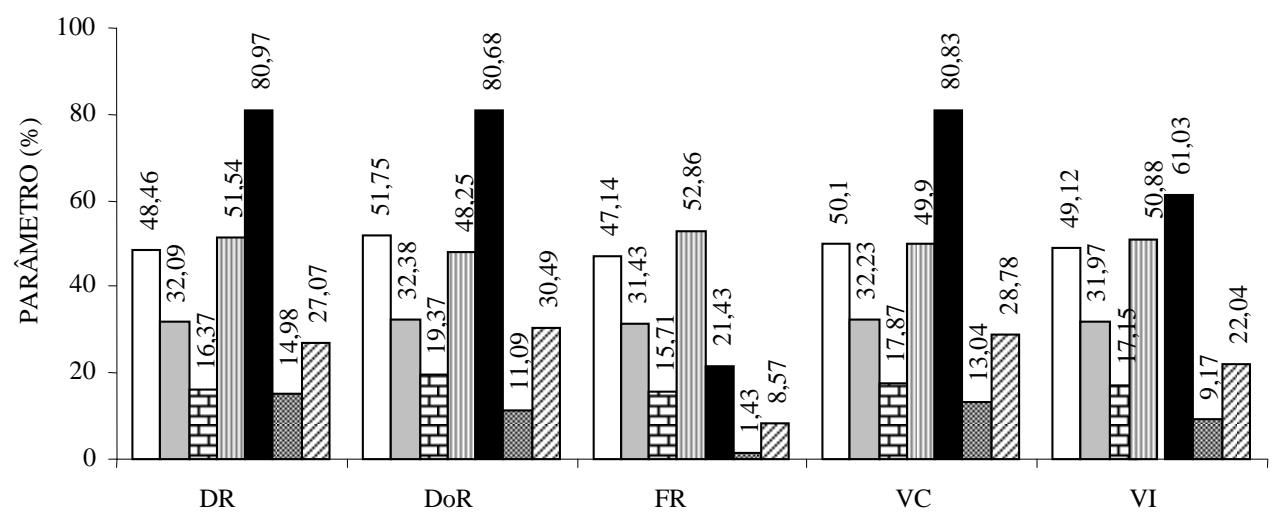

$\square$ Espécies Comerciais

口 Espécies Não Comerciais

/ 6 Comerciais+Importantes $\square$ Comerciais para Serraria

- 15 > Valor de Importância
日 Comerciais para Laminação

圆 Micropholis guyanensis

Figura 1. Valores percentuais apresentados pelas variáveis na estrutura horizontal, por grupos de espécies. $\mathrm{DR}=$ Densidade relativa; DoR $=$ Dominância relativa; $F R=$ Frequência relativa; $\mathrm{VC}=$ Valor de cobertura; $\mathrm{VI}=$ Valor de importância.

Figure 1. Presented percentual values by the horizontal structure variables, by group of species. DR = Relative density; DoR = Relative dominancy; FR = Relative frequency; $\mathrm{VC}=$ Values of covering; $\mathrm{VI}=$ Importance value.

Tamanhos de parcelas para estimar valores de cobertura

Acuracidade das estimativas

Os erros reais mostraram estimativas dos valores paramétricos variando de 5,9\% até 35,7\% para os grupos de espécies mais importantes, tanto fitossociologicamente (15 espécies) como comercialmente (6 espécies), principalmente a $10 \%$. 
Para as 15 espécies fitossociologicamente mais importantes, os melhores resultados foram obtidos pelas parcelas de $400 \mathrm{~m}^{2}$ nas dimensões de $40 \mathrm{~m}$ x $10 \mathrm{~m}$ e $20 \mathrm{~m}$ x $20 \mathrm{~m}$, com erros reais de $5,9 \%$ e $9,8 \%$. Já para as 6 espécies mais importantes comercialmente, o menor erro se deu para a parcela de 400 $\mathrm{m}^{2}(7,8 \%)$ nas dimensões de $40 \mathrm{~m} \times 10 \mathrm{~m}$ pela amostragem aleatória com intensidade amostral de $10 \%$. A Figura 2 mostra a distribuição dos erros reais para esses dois grupos de espécies. De maneira geral, os maiores erros reais foram encontrados nas parcelas de maiores dimensões.

A construção do gráfico mostrado na figura 2 baseou-se nas séries dos erros reais dos valores de cobertura, de forma crescente, na intensidade amostral de $10 \%$, por apresentar maior proximidade com os valores paramétricos, tanto no processo aleatório como no sistemático, facilitando a sua distinção visual.
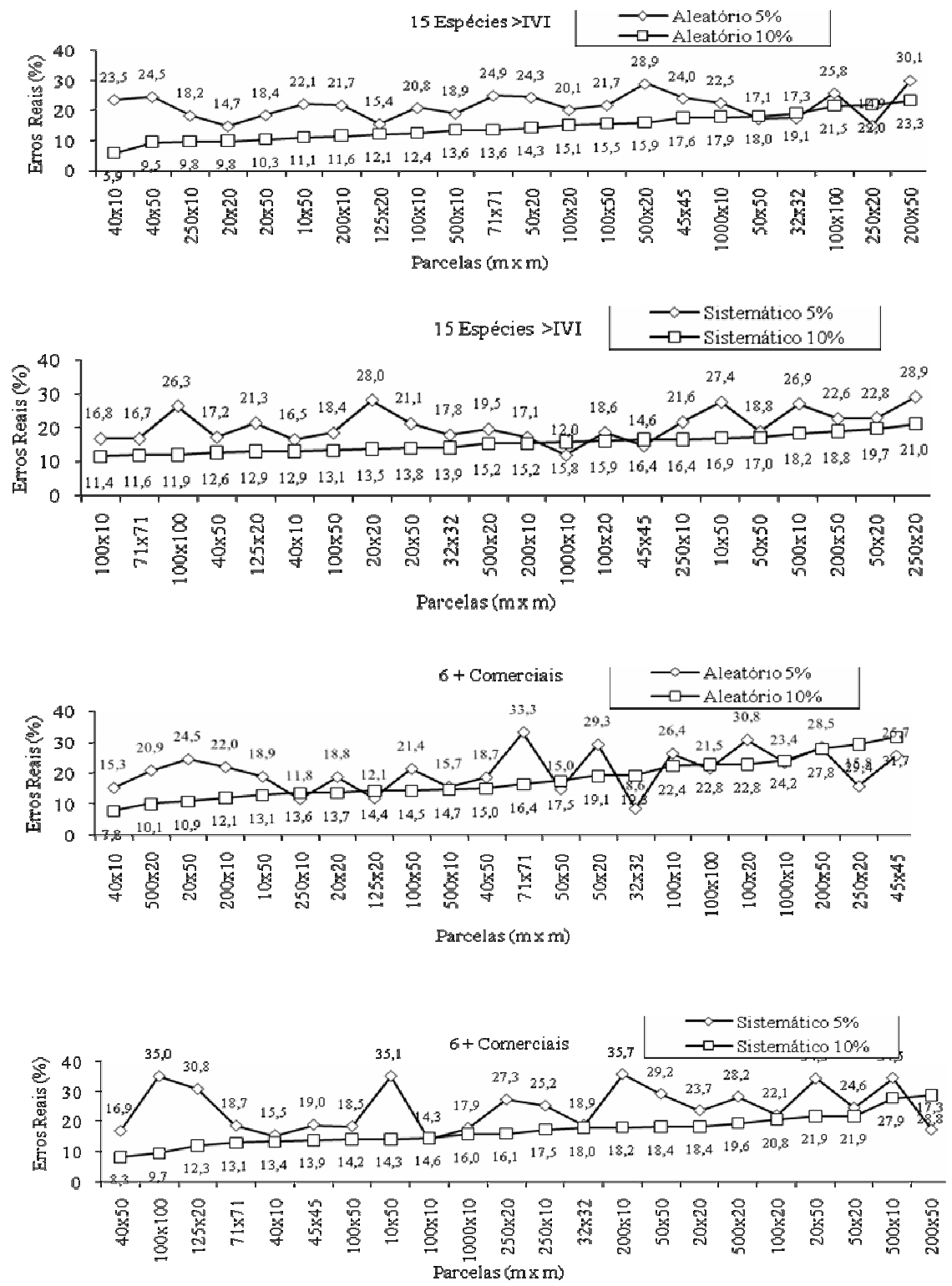

Figura 2. Erros reais por processos e intensidades amostrais, e por tamanhos e formas de parcelas para estimar valores de cobertura.

Figure 2. Real errors by sampling processes and intensities and by plot sizes and forms to estimate values of covering. 
Para todas as espécies, para os outros três grupos de espécies e para a espécie de maior importância na área, os erros reais sempre foram superiores a $40 \%$, demonstrando a inviabilidade de utilização dos dois processos amostrais e nos níveis testados neste trabalho. Maiores detalhes podem ser encontrados em Ubialli (2007). Dessa forma, observa-se que o uso de amostragem deve ser facultado apenas para os dois grupos que englobam o conjunto das espécies mais importantes fitossociologicamente e o das 6 espécies mais importantes comercialmente na área. Por outro lado, comprova-se que os altos erros provocados nas estimativas para os demais grupos de espécies se devem à grande heterogeneidade existente, tanto na abundância da grande maioria das espécies, consideradas "raras", como na sua dominância.

Ressalte-se a importância de se considerar a validade de se comparar resultados fitossociológicos, mesmo em áreas de mesma tipologia, com diferentes tamanhos de áreas e de critérios de inclusão de árvores, pelos seus diâmetros mínimos, como já observados por Isernhagen (2001).

\section{Influências da intensidade e do processo de amostragem}

De maneira geral, na medida em que se aumenta o tamanho da amostra, diminuem-se os erros de estimativa. Isso ocorreu, em média, em $83,1 \%$ das parcelas, cujos erros reais foram calculados para uma fração amostral de $10 \%$, enquanto que em apenas $16,9 \%$ das unidades de amostra testadas se obteve erros considerados adequados na intensidade amostral de 5\%. É importante ressaltar que, como erros adequados, foram considerados os erros de até $10 \%$.

Para todas as espécies e para as espécies comerciais, a maior intensidade amostral é responsável pelos menores erros reais em $91 \%$ dos tamanhos de parcelas testados. Índices próximos foram obtidos para os grupos de espécies não comerciais e para as 15 espécies fitossociologicamente mais importantes, com $86 \%$.

O processo de amostragem sistemático mostrou ser mais apropriado para estimar os erros reais em $52,6 \%$ das parcelas testadas. Os melhores índices percentuais foram obtidos para todas as espécies, $59,1 \%$, e para os grupos de espécies comerciais e comerciais para laminação, com 68,18 e 63,6\%, respectivamente. O processo de amostragem aleatório, por outro lado, foi mais preciso para o grupo de espécies para "serraria", para os 6 mais importantes comercialmente e para as 15 espécies fitossociologicamente mais importantes, com índices de 59,1\% para as duas primeiras e de 54,6\% para a última.

Como, pelos erros reais, somente para os grupos de espécies mais importantes fitossociologicamente e economicamente as estatísticas por amostragem são recomendadas, o processo de amostragem mais adequado para inferenciar os valores paramétricos é o aleatório, e a intensidade de amostragem é de $10 \%$, conforme se observa na tabela 5 .

Tabela 5. Percentuais de parcelas com erros reais adequados por intensidade e processos de amostragem para os valores de cobertura.

Table 5. Percentual of plots with adequate real errors by simpling processes and intesities for the values of covering.

\begin{tabular}{lcccc}
\hline \multirow{2}{*}{ Grupos de espécies } & \multicolumn{4}{c}{$\%$ de Parcelas } \\
\cline { 2 - 5 } & \multicolumn{2}{c}{ Intensidade amostragem } & \multicolumn{2}{c}{ Processo amostral } \\
& $\mathbf{5 \%}$ & $\mathbf{1 0 \%}$ & Aleatória & Sistemática \\
\hline Todas as Espécies & 9,09 & 90,91 & 40,91 & 59,09 \\
Comerciais & 13,64 & 86,36 & 31,82 & 68,18 \\
Com. Serraria & 18,18 & 81,82 & 59,09 & 40,91 \\
Com. Laminação & 22,73 & 77,27 & 36,36 & 63,64 \\
Com. 6 + Importantes & 27,27 & 72,73 & 59,09 & 40,91 \\
Não Comerciais & 13,64 & 86,36 & 50,00 & 50,00 \\
15 > VC & 13,64 & 86,36 & 54,55 & 45,45 \\
\hline Média & 16,88 & 83,12 & 47,40 & 52,60 \\
\hline
\end{tabular}




\section{CONCLUSÕES}

A família mais representativa da área estudada é Fabaceae, seguindo-se Apocynaceae e Sapotaceae. Micropholis guyanensis é a espécie mais importante fitossociologicamente. Possuem, também, presença marcante na área as famílias Moraceae, Lauraceae e Burseraceae.

O padrão de distribuição espacial da maioria das espécies resulta em aleatório quando parcelas menores $\left(400 \mathrm{~m}^{2}\right)$ são empregadas e se torna agregado quando as dimensões das parcelas são maiores (1 ha), demonstrando que parcelas maiores devem ser utilizadas para se obter o real padrão de distribuição espacial das espécies na área.

Para estudos fitossociológicos, o processo aleatório, com intensidade amostral de 10\%, mostrou estimativas bastante acuradas nos grupos de espécies mais importantes econômica e fitossociologicamente, principalmente para parcelas retangulares de $400 \mathrm{~m}^{2}$ e para $2.500 \mathrm{~m}^{2}$. Para os demais grupos, processos estimativos são desaconselhados.

O método de área fixa, com parcelas retangulares de $2.500 \mathrm{~m}^{2}$, produz estimativas acuradas para estudos fitossociológicos quando se consideram os grupos de espécies mais importantes do ponto de vista econômico e fitossociológico, independentemente do processo amostral, mas sobretudo para a intensidade amostral de $10 \%$.

\section{REFERÊNCIAS}

BARROS, P. L. C.; MACHADO, S. A. Aplicação de índices de dispersão em espécies de florestas tropicais da Amazônia brasileira. Curitiba: FUPEF, 1984, 43 p. (Série Científica, n. 1).

BENTES-GAMA, M. M.; SCOLFORO, J. R. S.; GAMA, J. R. V.; OLIVEIRA, A. D de. Estrutura e valoração de uma floresta de várzea alta na Amazônia. Cerne, v. 8, n. 1, p. 88-102, 2002.

BONETES, L. Tamanho de parcelas e intensidade amostral para estimar o estoque e índices fitossociológicos em uma Floresta Ombrófila Mista. Dissertação (Mestrado em Ciências Florestais) Setor de Ciências Agrárias, Universidade Federal do Paraná, Curitiba, 2004.

BRASIL. Ministério das Minas e Energia. Departamento Nacional de Produção Mineral. Projeto RADAMBRASIL. Volume 22 Folha SC 22 - Tocantins. Rio de Janeiro: Departamento Nacional da Produção Mineral, 1981.

BROWER, J.; ZAR, J. H. Field and laboratory methods for general ecology. 2.ed. Dubuque: Wm. C. Brown, 1984. $226 \mathrm{p}$.

DAUBENMIRE, R. Plant communities - a textbook of plant synecology. New York: Harper \& Row, 1968. $300 \mathrm{p}$.

FERREIRA, L. A.; BRAZ, E. M. Avaliação do potencial de extração e comercialização do óleo-resina de copaíba (Copaifera spp.) [on line] Disponível em: 〈http://www.nybg.org/bsci/acre/evaluation.html > . Acesso em: 14/06/2004.

GOMES, A. P.; SOUZA, A. L.; MEIRA NETO, J. A. A. Alteração estrutural de uma área de floresta explorada convencionalmente na Bacia do Paraíba do Sul, Minas Gerais, nos domínios de Floresta Atlântica. Revista Árvore, Viçosa, MG, v. 28, n. 3, p. 407-417, 2004.

GUAPYASSÚ, M. S. dos. Caracterização fitossociológica de três fases sucessionais e uma Floresta Ombrófila Densa. 195 f. Dissertação (Mestrado em Ciências Florestais) - Setor de Ciências Agrárias, Universidade Federal do Paraná, Curitiba, 1994.

HIGUCHI, N.; SANTOS, J.; JARDIM, F. C. S. Tamanho de parcela amostral para inventários florestais. Revista Silvicultura, São Paulo, v. 28, p. 649-656, 1982. (Anais do $4^{\circ}$ Congresso Florestal Brasileiro).

HUSCH, B. Planing a Forest Inventory. Rome: FAO, 1971. 120 p. (FAO Forest Products Studies, n. 17).

HUSCH, B.; MILLER, C. I.; BEERS, T. W. Forest Mensuration. 2. ed. New York, The Ronald Press Company, 1972. $410 \mathrm{p}$. 
INSTITUTO BRASILEIRO DE MEIO AMBIENTE E DOS RECURSOS NATURAIS RENOVÁVEIS (IBAMA). Diretoria de Incentivo a pesquisa e divulgação. Laboratório de Produtos Florestais. Catálogo de árvores do Brasil. Brasília, 2001, 896 p.

ISERNHAGEN, I. A Fitossociologia florestal do Paraná e os programas de recuperação de áreas degradadas: Uma Avaliação. 219 f. Dissertação (Mestrado em Botânica) - Setor de Ciências Biológicas, Universidade Federal do Paraná, Curitiba, 2001

KAGEYAMA, P.; GANDARA, F. B. Dinâmica de populações de espécies arbóreas: implicações para o manejo e a conservação. In: SIMPÓSIO DE ECOSSISTEMAS DA COSTA BRASILEIRA, 3., 1993, São Paulo. Anais... São Paulo: ACIESP, 1993. p. 12.

LEITÃO FILHO, H. F. Aspectos taxonômicos das florestas do estado de São Paulo. Silvicultura em São Paulo, São Paulo, 16A, n. 1, p. 197-206, 1982.

LONGHI, S. J. Agrupamento e análise fitossociológica de comunidades florestais na subbacia hidrográfica do rio Passo Fundo - RS. 198 f. Tese (Doutorado em Ciências Florestais) - Setor de Ciências Agrárias, Universidade Federal do Paraná, Curitiba, 1997.

MACHADO, S. A. Complete enumeration forest inventory versus cluster sampling methods applied in the Amazonic Rain Forest. Floresta, Curitiba, v. 18, n. 1/2, p. 122-130, jun./dez. 1988.

MARTINS, F. R. O método de quadrantes e a fitossociologia de uma floresta residual do interior do estado de São Paulo. 239 p. Tese (Doutorado) - Instituto de Biociências, universidade de São Paulo, São Paulo, 1979.

MARTINS, F. R. Estrutura de uma floresta mesófila. Campinas: UNICAMP, 1993. 246 p.

MARTINS, S. S.; COUTO, L.; MACHADO, C. C.; SOUZA, A. L. Efeito da exploração florestal seletiva em uma floresta estacional semidecidual. Revista Árvore, Viçosa, MG, v. 27, n. 1, p. 65-70, 2003.

MATTEUCCI, S. D.; COLMA, A. Metodologia para el estúdio de la vegetation. Washington: The General Secretarial of the Organization of American States, 1982. 167 p. (Série Biologia Monografia, 22).

MELLO, J. M. Análise comparativa de procedimentos amostrais em um remanescente de floresta nativa no município de Lavras, MG. 99 p. Dissertação (Mestrado em Engenharia Florestal) Universidade Federal de Lavras, Lavras, MG, 1995.

MUELLER DOMBOIS, D.; ELLENBERG, H. Aims and methods of vegetation and ecology. New York: J. Wiley \& Sons, 1974. 547 p.

PAULA, A.; SILVA, A. F.; SOUZA, A. L.; SANTOS, F. A. M. Alterações florísticas ocorridas num período de quatorze anos na vegetação arbórea de uma floresta estacional semidecidual em Viçosa-MG. Revista Árvore, Viçosa, MG, v. 26, n. 6, p. 743-749, 2002.

PÉLLICO NETO, S.; BRENA, D. A. Inventário florestal. Curitiba, 1997. 316 p.

PIELOU, E. C. An introduction to mathematical ecology. Nueva York: Willey Interscience,. 1969. 286 p.

PIELOU, E. C. Ecologycal diversity, Nueva York: Willey Interscience,. 1975. 165 p.

PIRES, J. M.; DOBZHANSKY, T.; BLACK, G. A. An estimate of the number of species to trees in an Amazonian forest community. Botanical Gazette, Chicago, n. 114, p. 467-77, 1953.

ROCHA, F. T. Levantamento florestal na Estação Ecológica dos Caetetus como subsídio para laudos de desapropriação ambiental. 2003. 156 f. Dissertação (Mestrado em Ciências Florestais) Escola Superior de Agricultura "Luiz de Queiroz", Piracicaba, 2003.

RONDON NETTO, R. M.; BOTELHO, S. A.; FONTES, M. A. L.; DAVID, A. C.; FARIA, J. M. R. Estrutura e composição florística da comunidade arbóreo-arbustiva de uma clareira de origem antrópica, em uma Floresta Estacional Semidecídua Montana, Lavras, MG. Revista Cerne, Lavras, v. 6, n. 2, p. 7984, 2000. 
SHEPHERD, G. S. Fitopac 1: Manual do usuário. Campinas: Universidade Estadual de Campinas, 1996. 80 p.

SILVA, J. N. M. Eficiência de diversos tamanhos e formas de unidades de amostra aplicadas em inventário florestal na região do baixo Tapajós. 83 f. Dissertação (Mestrado em Ciências Florestais) Setor de Ciências Agrárias, Universidade Federal do Paraná, Curitiba, 1980.

SILVA, L, A.; SCARIOT, A. Composição e estrutura da comunidade arbórea de uma Floresta Estacional Decidual sobre afloramento calcário no Brasil Central. Revista Árvore, Viçosa, MG, v. 28, n. 1, p. 6975, 2004.

SILVA JÚNIOR, M. C. Comparação entre matas de galeria no Distrito Federal e a efetividade do Código Florestal na proteção de sua diversidade arbórea. Acta Botanica Brasílica, Porto Alegre, v. 15, n. 1, p. 139-146, 2001.

SOUZA, A. L.; SCHETTINO, S.; JESUS, R. M. Dinâmica da regeneração natural em uma Floresta Ombrófila Densa Secundária, após corte de cipós, Reserva Natural da Companhia Vale do Rio Doce S.A., Espírito Santo, Brasil. Revista Árvore, Viçosa, MG, v. 26, n. 4, p. 411-419, 2002a.

SOUZA, A. L.; SCHETTINO, S.; JESUS, R. M. Dinâmica da composição florística de uma Floresta Ombrófila Densa Secundária, após corte de cipós, Reserva Natural da Companhia Vale do Rio Doce S.A., Espírito Santo, Brasil. Revista Árvore, Viçosa, MG, v. 26, n. 5, p. 549-558, 2002 b.

SPURR, S. H. Forest Inventory. New York: Ronald Press, 1952. 476 p.

UBIALLI, J. A. Comparação de métodos e processos de amostragem para estudos fitossociológicos e estimativas de estoque de uma Floresta Ecotonal na região norte matogrossense. $261 \mathrm{f}$. Tese (Doutorado em Ciências Florestais) - Setor de Ciências Agrárias, Universidade Federal do Paraná, Curitiba, 2007. 